\title{
A novel variant of androgen receptor is associated with idiopathic azoospermia
}

\author{
LISHA MOU ${ }^{1,2}$ and YAOTING GUI ${ }^{2}$ \\ ${ }^{1}$ Shenzhen Domesticated Organ Medical Engineering Research and Development Center, \\ Shenzhen Second People's Hospital, First Affiliated Hospital of Shenzhen University, Shenzhen, \\ Guangdong 518035; ${ }^{2}$ Guangdong and Shenzhen Key Laboratory of Male Reproductive Medicine and Genetics, \\ Institute of Urology, Peking University Shenzhen Hospital, Biomedical Research Institute, \\ Shenzhen PKU-HKUST Medical Center, Shenzhen, Guangdong 518036, P.R. China
}

Received June 19, 2015; Accepted March 7, 2016

DOI: $10.3892 / \mathrm{mmr} .2016 .5587$

\begin{abstract}
A variety of genetic variants can lead to abnormal human spermatogenesis. The androgen receptor (AR) is an important steroid hormone receptor that is critical for male sexual differentiation and the maintenance of normal spermatogenesis. In the present study, each exon of AR in 776 patients diagnosed with idiopathic azoospermia (IA) and 709 proven fertile men were sequenced using use panel re-sequencing methods to examine whether AR is involved in the pathogenesis of IA. Two synonymous variants and seven missense variants were detected. Of the missense variants, a luciferase assay demonstrated that the R630W variant reduced the transcriptional regulatory function of AR. This novel variant (p. R630W) of AR is the first to be identified in association with IA, thereby highlighting the importance of AR during spermatogenesis.
\end{abstract}

\section{Introduction}

Infertility is experienced by $10-15 \%$ of couples. It has previously been estimated that half of these cases are due to male infertility $(1,2)$, a serious form of which is idiopathic

Correspondence to: Dr Lisha Mou, Shenzhen Domesticated Organ Medical Engineering Research and Development Center, Shenzhen Second People's Hospital, First Affiliated Hospital of Shenzhen University, 3002 West Sungang Road, Shenzhen, Guangdong 518035, P.R. China

E-mail: molly__molly@163.com

Dr Yaoting Gui, Guangdong and Shenzhen Key Laboratory of Male Reproductive Medicine and Genetics, Institute of Urology, Peking University Shenzhen Hospital, Biomedical Research Institute, Shenzhen PKU-HKUST Medical Center, 1120 Lianhua Road, Shenzhen, Guangdong 518036, P.R. China

E-mail: guiyaoting2007@aliyun.com

Key words: male infertility, idiopathic azoospermia, androgen receptor, variant azoospermia (IA). Genetic analysis of patients with IA have revealed a variety of causes, predominantly chromosome aberrations or variants of functional genes associated with spermatogenesis $(3,4)$. Understanding the genetic variants may contribute to the diagnosis and management of infertility in men, and is crucial to prevent the passing of genetic defects to offspring in future generations via in vitro fertilization procedures.

Androgen receptor (AR) is an important steroid hormone receptor that is critical during male sexual differentiation and for the maintenance of normal spermatogenesis (5-7). AR belongs to a family of nuclear transcription factors that mediate the action of steroid hormones (8). There are 4 domains in the AR protein structure, including the $\mathrm{N}$-terminal transactivation domain, DNA-binding domain, hinge region and carboxyl ligand-binding domain $(9,10)$. Binding of androgens, including testosterone and $5 \alpha$-dihydrotestosterone, to the ligand-binding domain of AR results in nuclear translocation, where it acts as a transcriptional regulator (10).

Previously, a number of AR mutations or polymorphisms that cause or are associated with a spectrum of hereditary disorders, including complete and partial androgen insensitivity syndrome (CAIS and PAIS, respectively), were identified $(11,12)$. In order to identify variants of the AR gene in patients with IA, the present study sequenced each exon of AR in 776 patients diagnosed with IA and 709 proven fertile men. The results may be important for the accurate diagnosis of IA and useful for genetic counseling.

\section{Materials and methods}

Ethical approval. The present study was approved by the ethics committee of Peking University Shenzhen Hospital (Shenzhen, China; approval no. 20090018). The current study was approved on July 18, 2009, initiated on August 1, 2009 and terminated on December 1, 2014.

Patient samples. The patient inclusion criteria was the same as described previously (13), with certain modifications. A total of 1,880 azoospermic patients were recruited for the present study from the Center of Reproductive Medicine, 
Tongji Medical College, Huazhong University of Science and Technology (Wuhan, China) between January 2007 and October 2011. Among the subjects, 776 patients fulfilled the following criteria for IA diagnosis: i) No sperm detected in the pellets of semen samples on three different occasions; ii) no obstruction, inflammation or injury of the reproductive system or pelvic cavity; and iii) no karyotypic abnormality or Y chromosome microdeletion. A total of 709 fertile men from the Center of Physical Examination, Peking University Shenzhen Hospital were recruited as controls. Each had fathered at least one child without assisted reproductive techniques, including in vitro fertilization, intracytoplasmic sperm injection or intracytoplasmic morphologically selected sperm injection. Following panel re-sequencing and quality control steps, 776 patients aged 24-46 years (mean, 30.6 years) and 709 fertile men aged 29-51 years (mean, 35.6 years) were available for further analysis. Informed written consent was obtained from each subject.

Panel re-sequencing. Panel re-sequencing was performed as described previously (13). Genomic DNA samples $(5 \mu \mathrm{g})$ isolated from peripheral blood samples using the E.Z.N.A. Blood DNA kits (Omega Bio-Tek, Inc., Norcross, GA, USA) were sent to the Beijing Genomics Institute (Shenzhen, China) for exome capture and sequencing. The capture procedure was performed in solution with a NimbleGen custom array (Roche Applied Science, Madison, WI, USA) that enriched the exonic sequences of 654 infertility- or subfertility-associated genes. The majority these genes have been reviewed by Matzuk and Lamb (14). Additionally, the present study selected other genes that have been previously demonstrated to cause male reproductive defects in mouse models in studies published between November 2008 and December 2010 (15). Panel re-sequencing was performed using the Illumina platform (Illumina, Inc., San Diego, CA, USA) with 90 bp pair-end reads.

FASTQ sequence files were aligned against the human reference genome (NCBI build 37.1, hg19) with Short Oligonucleotide Analysis Package aligner software (version 2.21; www.soap.genomics.org.cn). Duplicated paired-end reads were removed from the merged data sets. Single nucleotide variants that were different from the hg19 reference genome were filtered out if they met any of the following criteria: i) A Phred-like quality score of $\leq 20$; ii) overall depth of $\leq 8 x$; estimated copy number of $\geq 2$; iii) or genomic distance between two adjacent variants of $<5 \mathrm{bp}$. In addition, the quality score of the major and minor allele at heterozygous loci were $\geq 20$. The variants were then annotated using an in-house functional prediction tool, and were compared with the dbSNP Build 135 (www.ncbi.nlm.nih.gov/projects/SNP/) and 1000 Genomes (www.1000genomes.org; as of August 2010). Validation of novel missense variants by Sanger sequencing. To validate the novel missense variants identified by deep sequencing, polymerase chain reaction (PCR) amplifications were performed and the PCR products were sequenced in both directions with a 3730 DNA Analyzer (Applied Biosystems; Thermo Fisher Scientific, Inc., Waltham, MA, USA). The primers for PCR and Sanger sequencing validation of the AR gene are presented in Table I.
Validation of novel missense variants by Sanger sequencing. As described in Panel re-sequencing, genomic DNA samples $(5 \mu \mathrm{g})$ were isolated from peripheral blood samples using the E.Z.N.A. Blood DNA kits (Omega Bio-Tek, Inc.). PCR was performed in a volume of $50 \mu \mathrm{l}$ containing $1 \mu \mathrm{M}$ of each forward and reverse primer, 50 ng DNA, and $25 \mu 1$ EmeraldAmp PCR Master mix (Takara Bio Inc., Otsu, Japan). Products were amplified in a thermocycler (MyCycler; Bio-Rad, Hercules CA, USA) with the following conditions: 30 cycles of $10 \mathrm{sec}$ at $98^{\circ} \mathrm{C}, 30 \mathrm{sec}$ at $60^{\circ} \mathrm{C}$, and $40 \mathrm{sec}$ at $72^{\circ} \mathrm{C}$. Amplicons were extracted from gels and sequenced in both directions with a 3730 DNA Analyzer (Applied Biosystems; Thermo Fisher Scientific, Inc.). The primers for PCR and Sanger sequencing validation of the AR gene are presented in Table I.

Protein alignment. Multiple protein alignments were performed with MegAlign 7.1.0 (DNASTAR, Inc., Madison, WI, USA). The amino acid sequences of the androgen receptor in humans, chimpanzees, rhesus monkeys, cows, rats, mice and chickens was determined. The identification numbers of androgen receptor protein were as follows: Human, NP_000035.2; chimpanzee, XP_009437511.1; rhesus, NP_001028083.1; cow, NP_001231056.1; rat, NP_036634.1; mouse, NP_038504.1; and chicken, NP_001035179.1).

Evaluation of coding single nucleotide polymorphisms. Sorting Intolerant From Tolerant (SIFT; sift.jcvi.org/) and PolyPhen 2.0 (genetics.bwh.harvard.edu/pph2/) analysis were used for the evaluation of coding single nucleotide polymorphisms $(16,17)$. SIFT is based on the premise that protein evolution is correlated with protein function. SIFT scores $\leq 0.05$ are predicted by the algorithm to be damaging, whereas scores $>0.05$ are considered tolerant. Predictions are based on a combination of phylogenetic, structural and sequence annotation information characterizing a substitution and its position in the protein. PolyPhen scores $>0.85$ are predicted by the algorithm to be probably damaging, scores $>0.15$ are considered possibly damaging, whereas scores $<0.15$ are considered benign.

Plasmid construction and site-directed mutagenesis. A human AR expression plasmid was provided as a gift from Dr Chawnshang Chang (George H. Whipple Lab for Cancer Research, Departments of Pathology and Urology, University of Rochester Medical Center, Rochester, NY, USA). Site-directed mutagenesis was performed to generate AR expression plasmids bearing the C290R, S495N or R630W variant, as described previously (13). DNA sequencing was performed to confirm the introduced variants. The PCR primers used for site-directed mutagenesis and plasmid construction are presented in Table II. Products were amplified in a thermocycler (Bio-Rad) using an Expand High Fidelity PCR system (Roche, Basel, Switzerland). The $50 \mu 1$ PCR reaction was conducted with $50 \mathrm{ng}$ templates, $1 \mu \mathrm{M}$ primer pairs, $200 \mu \mathrm{M}$ dNTPs and 2 units of DNA polymerase. The extension reaction was initiated by pre-heating the reaction mixture to $94^{\circ} \mathrm{C}$ for $3 \mathrm{~min} ; 16$ cycles of $94^{\circ} \mathrm{C}$ for $1 \mathrm{~min}, 52^{\circ} \mathrm{C}$ for $1 \mathrm{~min}$ and $68^{\circ} \mathrm{C}$ for $8 \mathrm{~min}$; followed by incubation at $68^{\circ} \mathrm{C}$ for $15 \mathrm{~min}$. DNA sequencing was conducted with a 3730 DNA Analyzer (Applied Biosystems) to confirm the introduced variants. 
Luciferase assay. Luciferase analysis was performed as described previously, with certain modifications (18). HeLa and TM4 cells (American Type Culture Collection, Manassas, VA, USA) were cultured in Dulbecco's modified Eagle's medium (Gibco; Thermo Fisher Scientific, Inc.) supplemented with $10 \%$ fetal bovine serum (Gibco; Thermo Fisher Scientific, Inc.), $100 \mathrm{U} / \mathrm{ml}$ penicillin (Gibco; Thermo Fisher Scientific, Inc.) and $100 \mu \mathrm{g} / \mathrm{ml}$ streptomycin (Gibco; Thermo Fisher Scientific, Inc.) at $37^{\circ} \mathrm{C}, 95 \%$ humidity and $5 \% \mathrm{CO}_{2}$ atmosphere. Cells were seeded in 24-well tissue culture plates for $24 \mathrm{~h}$ prior to transfection. Equivalent concentrations (100 ng) of wild-type (WT) or mutant AR expression plasmids were cotransfected with mouse mammary tumor virus (pMMTV) long terminal repeat plasmids (a gift from Dr Chawnshang Chang) into HeLa and TM4 cells using Lipofectamine 2000 (Invitrogen; Thermo Fisher Scientific, Inc.), according to the manufacturer's instructions. Cells were treated with or without $100 \mathrm{nM}$ testosterone (Sigma-Aldrich) after $6 \mathrm{~h}$ of transfection and harvested $24 \mathrm{~h}$ after treatment. Firefly and Renilla luciferase expression was assessed using a Dual-Luciferase Reporter Assay System (cat no. E1910; Promega Corporation, Madison, WI, USA). Renilla luciferase activity was normalized to that of firefly luciferase. The Modulus/ 9200-003 luminometer (Turner Biosystems, Sunnydale, CA, USA) was used in this study. Following normalization for transfection efficiency, induction factors were calculated as the ratio of the mean luciferase value for testosterone-stimulated versus non-testosterone-stimulated (ethanol vehicle-treated) samples.

Ultrasound examinations. All ultrasonographic measurements were conducted by the same ultrasonographer. Patients with novel AR variants were examined with scrotal ultrasonography to test the size of testes. All examinations were performed using ultrasound scanner (EUB-7500, Hitachi Medical Corporation, Tokyo, Japan) equipped with a linear array transducer (6.5 MHz; Model EUP-L65; Hitachi Medical Corporation).

Statistical analysis. All experiments were repeated at least three times. Data of the luciferase assay are expressed as the mean \pm standard deviation. SPSS version 17.0 statistical software (SPSS, Inc., Chicago, IL, USA) was used for statistical analysis. Analysis of variance (ANOVA) and Dunnett's t-test were used to compare the difference in the means between the variants and WT. $\mathrm{P}<0.05$ was considered to indicate a statistically significant difference. Data were log-transformed before ANOVA to satisfy the equal variances assumption.

\section{Results}

Identification of AR variant in patients with IA. To examine whether AR genetic defects were associated with IA, the present study screened for AR exonic variants in 776 patients with IA and 709 fertile men using massive parallel sequencing technology. As demonstrated in Table III, seven missense variants and two synonymous variants were detected in AR. Two of these missense variants (F827L and M887V) have been previously reported in the ExAC database. Another variant at amino acid 290 (C290W) has been previously described in the ExAC database, however, this variant was different from the variant identified in the present study (C290R). The three
Table I. Primers for polymerase chain reaction and Sanger sequencing validation of the AR gene.

Primer Sequence (5'-3')

\begin{tabular}{cc}
\hline $\begin{array}{c}\text { AR mut } 1 \\
\text { Forward } \\
\text { Reverse }\end{array}$ & CTCCGCTGACCTTAAAGACATCCT \\
AR mut2 & CTCGCCTTCTAGCCCTTTGGTGTA \\
Forward & CTCCCCATCCCCACGCTCGCATCA \\
Reverse & ATCCAGGGGCCCATTTCGCTTTTG \\
AR mut3 & \\
Forward & GGTTTAGCAGGTATTTGGGATGAT \\
Reverse & GAGTCGGGCTGGTTGTTGTC \\
\hline
\end{tabular}

$\mathrm{AR}$, androgen receptor.

Table II. Primers for polymerase chain reaction used for site-directed mutagenesis and plasmid construction.

\begin{tabular}{ll}
\hline Primer & \multicolumn{1}{c}{ Sequence (5'-3') } \\
\hline $\begin{array}{l}\text { hAR mut1 (T-C) } \\
\text { Forward }\end{array}$ & \\
TGCCCCATTGGCCGAA & cGCAAAGGTTCTCTGCT \\
Reverse & AGCAGAGAACCTTTGC \\
& gTTCGGCCAATGGGGCA \\
hAR mut2 (G-A) & \\
Forward & CTGGCGGGCCAGGAAA \\
Reverse & aCGACTTCACCGCACCT \\
& AGGTGCGGTGAAGTCG \\
hAR mut3 (C-T) & tTTCCTGGCCCGCCAG \\
Forward & \\
& ATGACTCTGGGAGCC \\
Reverse & AGGAAGCTGAAGAAACTT \\
& aGGCTCCCAGAGTCAT
\end{tabular}

Lower case bases indicate the mutant sites. AR, androgen receptor.

patient-specific missense variants (C290R, S495N and R630W) have not been previously reported in the dbSNP135 database, 1000 Genome Project dataset or ExAC database. These three novel patient-specific missense variants were further confirmed by Sanger sequencing (Fig. 1). Alignment of the amino acid sequence of AR to its orthologs in different species demonstrated that the R630W variant affected a highly conserved amino acid (Fig. 2). Based on this conservation behavior, the variant R630W was predicted to be possibly damaging to the protein, according to SIFT and PolyPhen-2.0 analysis (Table IV). The locations of these three missense variants within the AR protein are demonstrated in Fig. 3.

Effects of AR variants on AR function. To evaluate whether the identified patient-specific missense variants of AR inhibit its 
Table III. Androgen receptor variants and single nucleotide polymorphisms identified in patients with idiopathic azoospermia (IA; $n=776)$ and fertile controls $(n=709)$.

\begin{tabular}{|c|c|c|c|c|c|c|}
\hline Variant no. & Sequence variant & Amino acid change & IA patients, $n$ & Fertile controls, $n$ & Reported & Sample ID \\
\hline \multicolumn{7}{|l|}{ Missense } \\
\hline 1 & c. $868 \mathrm{~T}>\mathrm{C}$ & p.C290R & 1 & 0 & No & w320 \\
\hline 2 & c. $1484 \mathrm{G}>\mathrm{A}$ & p.S495N & 1 & 0 & No & w691 \\
\hline 3 & c. $1888 \mathrm{C}>\mathrm{T}$ & p.R630W & 1 & 0 & No & w530 \\
\hline 4 & c.569 C>T & p.T190I & 0 & 1 & No & 262 \\
\hline 5 & c. $616 \mathrm{~A}>\mathrm{G}$ & p.S206G & 0 & 1 & No & 285 \\
\hline 6 & c. $2481 \mathrm{C}>\mathrm{A}$ & p.F827L & 0 & 1 & Yes & 81 \\
\hline 7 & c. $2659 \mathrm{~A}>\mathrm{G}$ & p.M887V & 0 & 1 & Yes & 57 \\
\hline \multicolumn{7}{|c|}{ Synonymous } \\
\hline 8 & c. $639 \mathrm{G}>\mathrm{A}$ & None & 0 & 1 & Yes & 177 \\
\hline 9 & c. $1149 \mathrm{C}>\mathrm{T}$ & None & 2 & 0 & No & w325,w671 \\
\hline
\end{tabular}

regulatory function, luciferase reporter constructs containing the androgen responsive elements (MMTV promoter) were transfected into HeLa and TM4 cell lines. AR WT, C290R and $\mathrm{S} 495 \mathrm{~N}$, but not R630W variants, significantly increased MMTV promoter activity compared with empty vector in both cell types $(\mathrm{P}<0.05$; Fig. 4). These results indicate that $\mathrm{C} 290 \mathrm{R}$ and $\mathrm{S} 495 \mathrm{~N}$ did not affect the transcriptional regulation activity of AR. However, the transcriptional regulation activity of AR was inhibited by the R630W variant.

Clinical variable and hormone analysis. Scrotal color Doppler ultrasonography of the three patients with novel AR variants revealed small testes in the scrotal sac, with a homogeneous echotexture and wide hypoechogenicity. No solid or cystic lesions were observed. Table $\mathrm{V}$ summarizes the clinical and hormone data of these three patients, none of which had a family history of male infertility, CAIS or PAIS.

\section{Discussion}

Accumulating evidence indicates that AR is a ligand-dependent transcription factor that regulates the expression of androgen-responsive genes (19). Androgens and AR are essential in male spermatogenesis and fertility $(20,21)$.

Although the AR gene has $>700$ reported mutants and polymorphisms, only 5 mutants located in exon 1 have been observed in azoospermia patients with varying degrees of impaired spermatogenesis or mild AIS (22). In agreement with the present study, all previous subjects presented normal external genitalia, complicating the clinical diagnosis. Additionally, a previous study observed that male mice with total AR knockout exhibited female-typical external appearance, which was similar to human AIS or testicular feminization variant mice (23).

The current study sequenced the coding sequence of AR in a large group of patients with IA. The R630W variant was observed in 1 of the 776 patients with IA, but was not detected in any of the 709 fertile men sequenced or in other individuals previously reported in the public databases. The R630W variant was localized in the conserved

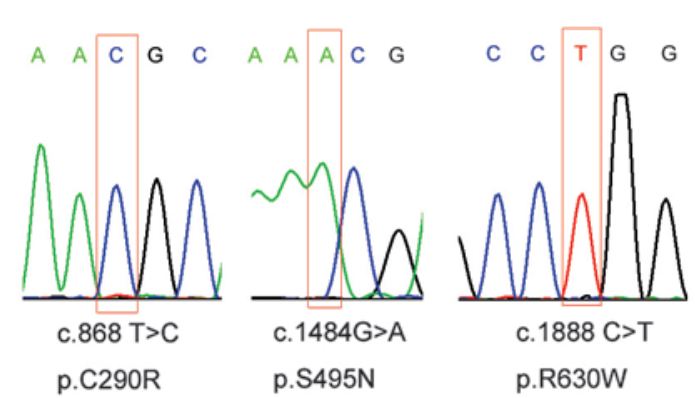

Figure 1. Three missense variants of the androgen receptor identified in patients with idiopathic azoospermia. Chromatogram traces from Sanger sequencing demonstrate the validated missense variants.

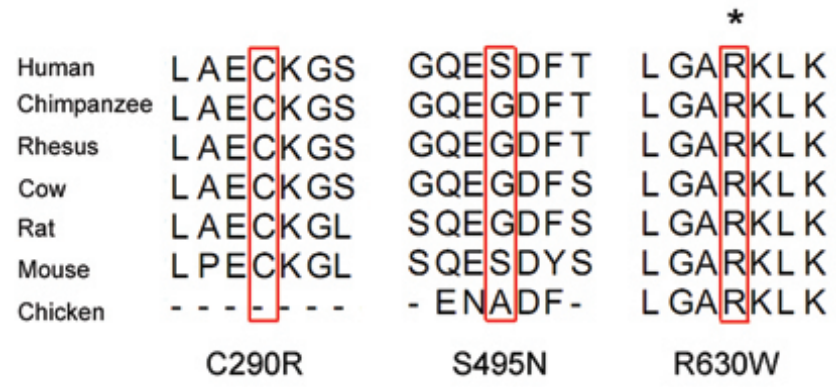

Figure 2. Evolutionary conservation of amino acids affected by the missense variants. Multiple protein alignments were performed with MegAlign. The identification numbers of androgen receptor protein were as follows: Human, NP_000035.2; chimpanzee, XP_009437511.1; rhesus, NP_001028083.1; cow, NP_001231056.1; rat, NP_036634.1; mouse, NP_038504.1; and chicken, NP_001035179.1). The variant alleles are boxed, and the * indicates the conserved residue.

DNA-binding domain of AR, which is in accordance with the abolished transcriptional activity of AR caused by this mutation. Computerized analysis using PolyPhen-2.0 and SIFT software classified the R630W variant as damaging, predicting it to have a deleterious effect on the protein structure. Additionally, local alignment analysis of the amino acid sequences of AR demonstrated that the affected arginine residue was highly conserved in multiple species, including 
Table IV. List of missense variants predicted to be functionally significant by SIFT and PolyPhen 2.0 programs.

\begin{tabular}{|c|c|c|c|c|c|}
\hline \multirow[b]{2}{*}{ Nucleotide change } & \multirow[b]{2}{*}{ Amino acid change } & \multicolumn{2}{|c|}{ SIFT $^{\mathrm{a}}$} & \multicolumn{2}{|c|}{ PolyPhen- $2.0^{\mathrm{b}}$} \\
\hline & & Score & Prediction & Score & Prediction \\
\hline c.868 T>C & p.C290R & 0.340 & Tolerated & 0.995 & Possibly damaging \\
\hline c. $1484 \mathrm{G}>\mathrm{A}$ & p.S495N & 0.480 & Tolerated & 0.066 & Benign \\
\hline c. $1888 \mathrm{C}>\mathrm{T}$ & p.R630W & 0.000 & Damaging & 1.000 & Probably damaging \\
\hline
\end{tabular}

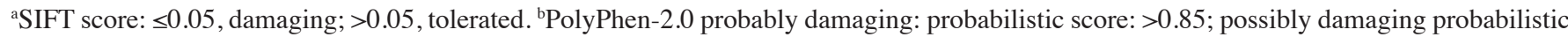
score: $>0.15$; and benign: probabilistic score: $<0.15$. SIFT, Sorting Intolerant From Tolerant.

Table V. Clinical and hormone profile of patients with idiopathic azoospermia with novel androgen receptor missense variants.

\begin{tabular}{lccccc}
\hline Sample ID & Age, years & FSH, IU/l & LH, IU/l & Estradiol, $\mathrm{pg} / \mathrm{ml}{ }^{\mathrm{c}}$ & Testosterone, $^{\mathrm{b}} / \mathrm{ml} \mathrm{l}^{\mathrm{d}}$ \\
\hline w320 & 25 & 27.19 & 14.51 & 29.64 & 4.23 \\
w530 & 30 & 8.12 & 11.46 & 66.61 & 8.75 \\
w691 & 24 & 25.18 & 8.40 & 20.59 & 3.15 \\
\hline
\end{tabular}

Normal ranges are ${ }^{\mathrm{a}} 1.5-12.5 \mathrm{IU} / \mathrm{l},{ }^{\mathrm{b}} 1.7-8.6 \mathrm{IU} / 1,{ }^{\mathrm{c}} 7.63-42.6 \mathrm{pg} / \mathrm{ml},{ }^{\mathrm{d}} 2.8-8.0 \mathrm{ng} / \mathrm{ml}$. FSH, follicle stimulating hormone; LH, luteinizing hormone.

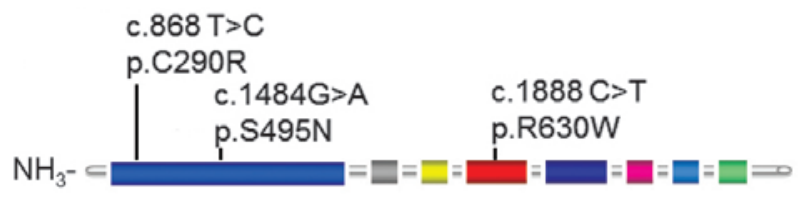

Figure 3. Variants identified in the androgen receptor (AR) gene. The positions of C290R, S495N and R630W variants are indicated on a modeled structure of the AR protein.

chickens. The evolutionary preservation of the entire region around this residue across multiple species indicates that variants in this region may affect the normal functions of the AR protein.

When evaluating the pathogenic effect of AR variants in patients with fertility issues, an important question to address is whether the variant affects transcriptional regulatory function of AR. The present study demonstrated that the R630W variant affected the transcriptional regulatory function of $\mathrm{AR}$ at the MMTV promoter.

The hormonal profile of the patient carrying the R630W mutation (w530) was different to the other two patients with novel AR variants. Compared with patients w320 and w691, patient w530 exhibited low follicle stimulating hormone, and high estradiol and testosterone. Thus, the R630W mutation potentially reduces the transcriptional activity of AR, which leads to an imbalance of the hormonal profile.

In conclusion, the current study identified seven missense variants and two synonymous novel variants of AR using massive parallel sequencing technology. Functional analysis confirmed that the R630W variant suppressed the normal transcriptional regulatory function of AR. These results suggested that AR was important in human spermatogenesis. This study also demonstrated that systematic analysis of the
A

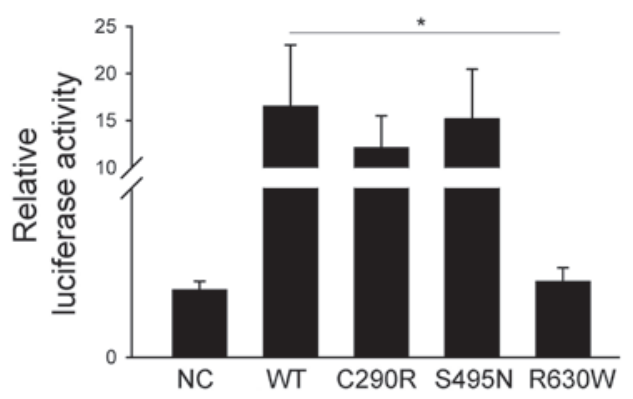

B

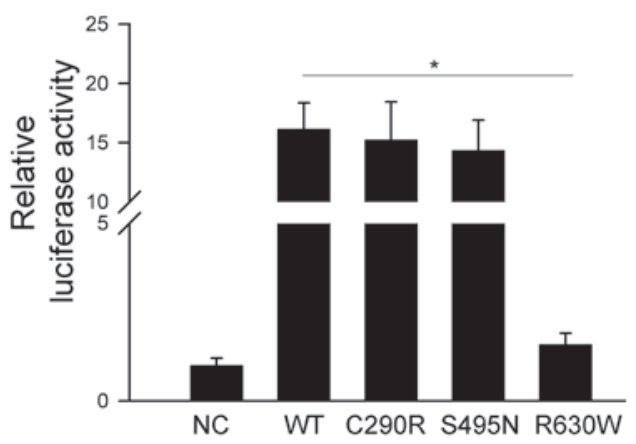

Figure 4. Effect of the androgen receptor (AR) variants on AR function. The WT or mutant AR expression vectors were cotransfected with testosterone-inducible pMMTV-LUC plasmid into (A) HeLa and (B) TM4 cells. NC plasmid (pcDNA3.1-HA) was cotrasfected with the pMMTV-LUC plasmid. Luciferase activities were measured with or without testosterone treatment. Compared with WT and other variants, the AR R630W variant did not increase pMMTV activity in the presence of testosterone. Results are presented as fold-change of testosterone- versus vehicle-treated samples. Values are presented as the mean \pm standard deviation. " $\mathrm{P}<0.05$. NC, negative control; WT, wild-type.

genetic mutations in large cohorts of patients complemented by subsequent functional assays may provide novel insights into the cause of IA in humans. 


\section{Acknowledgements}

The current study was supported by grants from the National Natural Science Foundation of China (grant nos. 31271244 and 81200465), Guangdong Natural Science Foundation of China (grant no. 2014A030313785), Shenzhen Foundation of Science and Technology (grant nos. GJHZ20140414170821192 and JCYJ20140414170821337), the 'Three Outstanding Projects' of Shenzhen, the Project of Shenzhen Engineering Center and the Key Laboratory Project of Shenzhen Second People's Hospital.

\section{References}

1. Jarvi K, Lo K, Fischer A, Grantmyre J, Zini A, Chow V and Mak V: CUA Guideline: The workup of azoospermic males. Can Urol Assoc J 4: 163-167, 2010.

2. Hirsh A: Male subfertility. BMJ 327: 669-672, 2003.

3. Alechine E and Corach D: High-throughput screening for spermatogenesis candidate genes in the $\mathrm{AZFc}$ region of the Y chromosome by multiplex real time PCR followed by high resolution melting analysis. PLoS One 9: e97227, 2014.

4. Xu M, Qin Y, Qu J, Lu C, Wang Y, Wu W, Song L, Wang S, Chen F, Shen $\mathrm{H}$, et al: Evaluation of five candidate genes from GWAS for association with oligozoospermia in a Han Chinese population. PLoS One 8: e80374, 2013.

5. Heemers HV and Tindall DJ: Androgen receptor (AR) coregulators: A diversity of functions converging on and regulating the AR transcriptional complex. Endocr Rev 28: 778-808, 2007.

6. Heinlein CA and Chang C: Androgen receptor (AR) coregulators: An overview. Endocr Rev 23: 175-200, 2002.

7. Patrão MT, Silva EJ and Avellar MC: Androgens and the male reproductive tract: An overview of classical roles and current perspectives. Arq Bras Endocrinol Metabol 53: 934-945, 2009.

8. Li CY, Earley RL, Huang SP and Hsu Y: Fighting experience alters brain androgen receptor expression dependent on testosterone status. Proc Biol Sci 281: 20141532, 2014.

9. Ferlin A, Bartoloni L, Rizzo G, Roverato A, Garolla A and Foresta C: Androgen receptor gene CAG and GGC repeat lengths in idiopathic male infertility. Mol Hum Reprod 10: 417-421, 2004.
10. Gottlieb B, Lombroso R, Beitel LK and Trifiro MA: Molecular pathology of the androgen receptor in male (in)fertility. Reprod Biomed Online 10: 42-48, 2005.

11. Bermúdez de la Vega JA, Fernández-Cancio M, Bernal S and Audí L: Complete androgen insensitivity syndrome associated with male gender identity or female precocious puberty in the same family. Sex Dev 9: 75-79, 2015.

12. Shao J, Hou J, Li B, Li D, Zhang N and Wang X: Different types of androgen receptor mutations in patients with complete androgen insensitivity syndrome. Intractable Rare Dis Res 4: 54-59, 2015.

13. Mou L, Wang Y, Li H, Huang Y, Jiang T, Huang W, Li Z, Chen J, Xie J, Liu Y, et al: A dominant-negative mutation of HSF2 associated with idiopathic azoospermia. Hum Genet 132: 159-165, 2013.

14. Matzuk MM and Lamb DJ: The biology of infertility: Research advances and clinical challenges. Nature Med 14: 1197-1213, 2008.

15. Li Z, Huang Y, Li H, Hu J, Liu X, Jiang T, Sun G, Tang A, Sun X, Qian W, et al: Excess of rare variants in genes that are key epigenetic regulators of spermatogenesis in the patients with non-obstructive azoospermia. Sci Rep 5: 8785, 2015.

16. Ng PC and Henikoff S: Predicting deleterious amino acid substitutions. Genome Res 11: 863-874, 2001.

17. Ramensky V, Bork P and Sunyaev S: Human non-synonymous SNPs: server and survey. Nucleic Acids Res 30: 3894-3900, 2002.

18. Mou L, Zhang Q, Wang Y, Zhang Q, Sun L, Li C, Huang W, Yuan Y, Duan Y, Diao R, et al: Identification of Ube2b as a novel target of androgen receptor in mouse sertoli cells. Biol Reprod 89: 32, 2013

19. Lu J, Van der Steen T and Tindall DJ: Are androgen receptor variants a substitute for the full-length receptor? Nat Rev Urol 12: 137-144, 2015.

20. Aquila S and De Amicis F: Steroid receptors and their ligands: Effects on male gamete functions. Exp Cell Res 328: 303-313, 2014.

21. Smith LB and Walker WH: The regulation of spermatogenesis by androgens. Semin Cell Dev Biol 30: 2-13, 2014.

22. Gottlieb B, Beitel LK, Nadarajah A, Paliouras M and Trifiro M: The androgen receptor gene mutations database: 2012 update. Hum Mutat 33: 887-894, 2012.

23. Yeh S, Tsai MY, Xu Q, Mu XM, Lardy H, Huang KE, Lin H, Yeh SD, Altuwaijri S, Zhou X, et al: Generation and characterization of androgen receptor knockout (ARKO) mice: An in vivo model for the study of androgen functions in selective tissues. Proc Natl Acad Sci USA 99: 13498-13503, 2002. 